Mon. Not. R. Astron. Soc. 000, 18 () Printed 8 March $2022 \quad$ (MN LATEX style file v2.2)

\title{
On the formation of planetary systems in photoevaporating transition discs
}

\author{
Caroline Terquem \\ Physics Department, University of Oxford, Keble Road, Oxford OX1 3RH, UK \\ Institut d'Astrophysique de Paris, UPMC Univ Paris 06, CNRS, UMR7095, 98 bis bd Arago, F-75014, Paris, France \\ E-mail: caroline.terquem@physics.ox.ac.uk
}

\begin{abstract}
In protoplanetary discs, planetary cores must be at least $0.1 \mathrm{M}_{\oplus}$ at 1 au for migration to be significant; this mass rises to $1 \mathrm{M}_{\oplus}$ at 5 au. Planet formation models indicate that these cores form on million year timescales. We report here a study of the evolution of $0.1 \mathrm{M}_{\oplus}$ and $1 \mathrm{M}_{\oplus}$ cores, migrating from about 2 and 5 au respectively, in million year old photoevaporating discs. In such a disc, a gap opens up at around 2 au after a few million years. The inner region subsequently accrete onto the star on a smaller timescale. We find that, typically, the smallest cores form systems of non-resonant planets beyond 0.5 au with masses up to about $1.5 \mathrm{M}_{\oplus}$. In low mass discs, the same cores may evolve in situ. More massive cores form systems of a few earth masses planets. They migrate within the inner edge of the disc gap only in the most massive discs. Delivery of material to the inner parts of the disc ceases with opening of the gap. Interestingly, when the heavy cores do not migrate significantly, the type of systems that are produced resembles our solar system. This study suggests that low mm flux transition discs may not form systems of planets on short orbits but may instead harbour earth mass planets in the habitable zone.
\end{abstract}

Key words: methods: numerical — accretion, accretion discs — planets and satellites: formation — planet-disc interactions — planetary systems

\section{INTRODUCTION}

Before the discovery of extrasolar planets, explaining the formation of terrestrial planets in our solar system was already a challenging task. The detection of a large variety of planetary systems containing Earth-mass or super-Earth planets by the Kepler satellite has made the task even more arduous. Terrestrial mass planets are usually considered to form either in situ or through inward migration. In situ formation was the early scenario proposed for forming planets in our solar system (Wetherill 1988, Lissauer 1993 and references therein), and has been put forward as a way to explain the Kepler candidates (Hansen \& Murray 2013). However, it has been argued that this model is not consistent with the distribution of solids in discs (Raymond \& Cossou 2014, Schlichting 2014). Also, in situ formation models have difficulties forming cores of giant planets before the gas dissipates (Thommes et al. 2003, Chambers 2016). Formation through inward migration is an efficient way of obtaining tight systems of super-Earths with short periods (Terquem \& Papaloizou 2007, Haghighipour 2013 and references therein), but fails to explain terrestrial planets similar to those in our solar system. However, migration of planets in dissipating discs has recently been studied (Coleman \&
Nelson 2014, Cossou et al. 2014, Coleman \& Nelson 2016) and seems to offer a way of combining the advantages of both models for forming terrestrial planets.

Coleman \& Nelson $(2014,2016)$ studied the evolution of systems containing initially 36 or 52 cores with masses of 0.3 or $0.1 \mathrm{M}_{\oplus}$, respectively, spread between 1 and $20 \mathrm{au}$. In addition, there were thousands of planetesimals with masses 10,20 or 50 times smaller than that of the embryos and distributed in between the planets. The disc had a surface gas density at least equal to that of the minimum solar mass nebula, i.e. $\Sigma \propto r^{-3 / 2}$ and $\Sigma=1.7 \times 10^{3} \mathrm{~g} \mathrm{~cm}^{-2}$ at $1 \mathrm{au}$, and was subject to photoevaporation and viscous evolution. They found that terrestrial-mass planets and super-Earths formed in the discs with the lowest masses. In the models with small abundance of solids and large planetesimals, growth was found to be limited so that migration was inefficient. Such models resulted in systems of low mass planets spread out through the disc and in which mean motion resonances were destroyed after the disc dissipated.

Cossou et al. (2014) started with cores with masses between 0.1 and $2 \mathrm{M}_{\oplus}$ spread between 1 and $20 \mathrm{au}$. The total mass in the cores was between 21 and $84 \mathrm{M}_{\oplus}$. They considered a disc with a surface gas density of $300 \mathrm{~g} \mathrm{~cm}^{-2}$ at 1 au and $\Sigma \propto r^{-1 / 2}$. In some of their simulations, the disc's mass 
was decreased exponentially to mimic disc's viscous evolution and photoevaporation. Systems of hot super-Earths in mean motion resonances were produced in discs which were not dissipating, whereas the systems were more spread out and not in resonances when dissipation was included.

In these studies, all types of planets formed from the same parent population and, in general, the planetary systems that were produced at around 1 au contained planets more massive than the terrestrial planets in our solar system. In this paper, we consider a model where different parent populations exist at different locations and the disc is photoevaporating.

In typical protoplanetary discs, the type-I migration timescale becomes comparable to the disc lifetime for cores at least as massive as 0.1 and $1 \mathrm{M}_{\oplus}$ at 1 and $5 \mathrm{au}$, respectively. Planet formation models suggest that it takes at least 1 Myr for $0.1 \mathrm{M}_{\oplus}$ cores to form at $\sim 1$ au. More massive cores, with a mass $\sim 1 \mathrm{M}_{\oplus}$, may form on this timescale beyond the snow line, at around $5 \mathrm{au}$. This prompts us to consider a model where the initial conditions are a population of $0.1 \mathrm{M}_{\oplus}$ cores between 1 and 5 au and a population of $1 \mathrm{M}_{\oplus}$ cores beyond $5 \mathrm{au}$, which start migrating when the disc is $\sim 1 \mathrm{Myr}-$ old. The total mass in these populations of cores is set by the initial surface density in the disc.

We evolve these initial populations in a disc which undergoes a transition due to $\mathrm{X}$-ray photoevaporation. Transition discs are defined as discs lacking emission in the nearinfrared, which means that there is a (large) hole in the dust distribution in their inner parts. Observations strongly suggest two types of transition discs: those with low $\mathrm{mm}$ flux, which have low accretion rates and hole sizes smaller than about $20 \mathrm{au}$, and those with high mm flux, which have higher accretion rates and hole sizes larger than 20 au (Owen \& Clarke 2012, Owen 2016 and references therein). It is believed that low mm flux transition discs are in the process of dispersing, whereas high mm flux transition discs are not. The most commonly accepted interpretation for low mm flux transition discs is X-ray photoevaporation. In this model, a gap opens up after about 3 Myr (75\% of the disc lifetime) at $\sim 2 \mathrm{au}$, where the accretion rate in the disc matches the photoevaporation rate (Owen, Ercolano \& Clarke 2011). The inner parts of the disc then become decoupled from the outer parts and cannot be resupplied in gas and dust. They subsequently accrete onto the central star whereas the outer edge of the gap recedes due to photoevaporation. By contrast, it is believed that, in high mm flux transition discs, a massive planet (with a mass of a few Jupiter masses) is responsible for creating a gap.

The above discussion suggests that, in low mm flux transition discs, $0.1 \mathrm{M}_{\oplus}$ and $1 \mathrm{M}_{\oplus}$ cores forming between 1 and $5 \mathrm{au}$ and beyond $5 \mathrm{au}$, respectively, on a timescale of $1 \mathrm{Myr}$, would start to migrate a million years or so before a gap opens up at around 2 au. As their migration timescale itself is on the order of a million years, it can be expected that the dispersion of the disc will prevent migration of cores to very small radii and also that the formation of the gap will prevent massive cores to be delivered to the region of terrestrial planets. This is the model we explore in this paper.

The plan of the paper is as follows. In section 2 we describe the disc evolution model and justify the parameters that are used in the simulations. We also give expressions for the planet migration and eccentricity damping timescales and briefly discuss core formation timescales. In section 3 , we present $N$-body simulations of cores migrating in photoevaporating discs. We first describe the numerical scheme and the initial set up. We then present the results of the simulations. We show that, for reasonable parameters that are consistent with the observations, a population of $0.1 \mathrm{M}_{\oplus}$ cores originating from between 2 and 4 au migrate down to 0.5-1 au. The final masses of planets are between a fraction of an Earth mass and $\sim 1.5 \mathrm{M}_{\oplus}$. As for the population of $1 \mathrm{M}_{\oplus}$ cores originating from $\sim 5 \mathrm{au}$, it forms a few cores of a few Earth masses which may migrate down below the inner edge of the gap only in the most massive discs considered here. In less massive discs, cores with a mass comparable to that of Jupiter may be left at a few au from the star. Finally, in section 4 we summarize and discuss our results.

\section{DISC EVOLUTION AND PLANET FORMATION}

In this section, we review the different timescales that are used in the simulations.

\subsection{Disc evolution and parameters}

We adopt an initial surface density profile in the disc:

$$
\Sigma(r, t=0)=\Sigma_{1}\left(\frac{r}{1 \mathrm{au}}\right)^{-1},
$$

where $\Sigma_{1}$ is the initial surface density at 1 au. This density gradient gives the best fit to the thermal continuum emission from $\sim 1$ Myr old discs in the Ophiuchus star-forming region, as shown by Andrews et al. (2010). We express $\Sigma_{1}$ in terms of the disc mass within $50 \mathrm{au}, M_{50}$ :

$$
\Sigma_{1}=2.8 \times 10^{4} \frac{M_{50}}{\mathrm{M}_{\odot}} \mathrm{g} \mathrm{cm}^{-2} .
$$

In this paper, we focus on the evolution of planetary systems in transition discs which have low mm fluxes and are supposed to be the consequence of $\mathrm{X}$-ray photoevaporation (Owen 2016 and references therein). In this model, a gap first opens up in the disc at the location where the accretion rate becomes equal to the photoevaporation rate. At this point, the parts of the disc within the inner edge of the gap become isolated from the outer parts, and cannot be resupplied in gas and dust. They accrete onto the central star on a timescale of a few $10^{5}$ years and become depleted. At the same time, the outer edge of the gap moves out because of erosion due to photoevaporation. The evolution of a disc subject to such a process has been calculated by Owen, Ercolano \& Clarke (2011). Here, we evolve the surface density profile $\Sigma(r, t)$ in such a way as to reproduce their calculation.

We start with $\Sigma(r, 0)$ given by equation (1). After a time $t=t_{\text {gap }}$ which is between 1 and $3 \mathrm{Myr}(\sim 75 \%$ of the disc lifetime, see Owen et al. 2011), a gap opens up between the radii that we fix to be $r_{10}=2 \mathrm{au}$ and $r_{20}=3 \mathrm{au}$. Between $t=0$ and $t_{\text {gap }}$, we assume that $\Sigma$ decreases linearly with time, i.e.: 


$$
\Sigma(r, t)=\Sigma(r, 0)\left[1-\frac{(1-\eta) t}{t_{\text {gap }}}\right], \text { for } 0 \leq t \leq t_{\text {gap }}
$$

where $\eta \equiv \Sigma\left(r, t_{\text {gap }}\right) / \Sigma(r, 0)$ is the fraction of mass left after a time $t_{\text {gap }}$. Subsequently, the inner disc disperses on a timescale $t_{\nu}$ such that:

$$
\begin{gathered}
\Sigma\left(r_{\text {cav }} \leq r \leq r_{10}, t\right)=\eta \Sigma(r, 0)\left[1-\frac{\left(t-t_{\text {gap }}\right)}{t_{\nu}}\right] \\
\quad \text { for } t_{\text {gap }} \leq t \leq t_{\text {gap }}+t_{\nu} \\
\Sigma\left(r \leq r_{10}, t\right)=0, \text { for } t \geq t_{\text {gap }}+t_{\nu},
\end{gathered}
$$

where $r_{\text {cav }}=0.05$ au if the radius of the inner disc cavity produced by the magnetic interaction between the disc and the star. We will take $t_{\nu} \sim 10^{4}-10^{5} \mathrm{yr}$ as this is the viscous timescale at 2 au in a standard $\alpha$-disc model (Shakura \& Sunyaev 1973) with $\alpha$ between $10^{-3}$ and 0.01 . After the gap opens up, its outer edge $r_{2}$ moves out according to:

$$
r_{2}=r_{20}+\left(10 \mathrm{au}-r_{20}\right) \frac{t-t_{\mathrm{gap}}}{3.2 \times 10^{6} \mathrm{yr}-t_{\mathrm{gap}}}, \text { for } t \geq t_{\mathrm{gap}}
$$

so that after $3.2 \times 10^{6}$ yr $(\sim 80 \%$ of the disc lifetime, see Owen et al. 2011), the outer edge has moved up to $10 \mathrm{au}$. The surface density does not vary significantly beyond $r_{2}$ :

$$
\begin{array}{r}
\Sigma\left(r \geq r_{2}, t\right)=\Sigma\left(r \geq r_{2}, t_{\text {gap }}\right)=\eta \Sigma\left(r \geq r_{2}, 0\right), \\
\text { for } t \geq t_{\text {gap }},
\end{array}
$$

while it is zero inside the gap:

$$
\Sigma\left(r_{10} \leq r \leq r_{2}, t\right)=0, \text { for } t \geq t_{\text {gap }}
$$

In the numerical simulations presented below, we will take $\Sigma_{1} \sim 10^{3} \mathrm{~g} \mathrm{~cm}^{-2}$ at $t=0$, i.e. $M_{50} \lesssim 0.1 \mathrm{M}_{\odot}$ initially. More massive discs would be gravitationally unstable. It has been pointed out that the observed range of accretion rates in $\mathrm{T}$ Tauri stars require an initial disc mass between 0.01 and $0.2 \mathrm{M}_{\odot}$ (Hartmann et al. 1998). Masses derived for the $\sim 1$ Myr old discs in the Ophiuchus star-forming region are on the order of $0.01 \mathrm{M}_{\odot}$ within 50 au (Andrews et al. 2010). This suggests that $\eta=0.1$ in equation (3). A decrease in disc's mass by a factor 10 during the first Myr of evolution is also consistent with the observations and modelling of discs around $\mathrm{T}$ Tauri stars in the Taurus and Chamaelon I molecular clouds complex (Hartmann et al. 1998).

The surface density at different times is shown in figure (1) for $t_{\text {gap }}=3 \mathrm{Myr}, t_{\nu}=0.2 \mathrm{Myr}$ and $\Sigma_{1}=2.7 \times$ $10^{3} \mathrm{~g} \mathrm{~cm}^{-2}$.

With the initial surface density of gas given by equation (1), and adopting a mass dust-to-gas ratio of 0.01 , we calculate that the initial mass of dust in an annulus between two radii $r_{\text {in }}$ and $r_{\text {out }}$ is:

$$
\begin{aligned}
M_{\text {dust }} & =2 \pi(1 \mathrm{au})^{2} \Sigma_{1}\left(\frac{r_{\mathrm{out}}}{1 \mathrm{au}}-\frac{r_{\mathrm{in}}}{1 \mathrm{au}}\right) \\
& \simeq 2.4 \mathrm{M}_{\oplus}\left(\frac{\Sigma_{1}}{10^{3} \mathrm{~g} \mathrm{~cm}^{-2}}\right)\left(\frac{r_{\mathrm{out}}}{1 \mathrm{au}}-\frac{r_{\mathrm{in}}}{1 \mathrm{au}}\right) .
\end{aligned}
$$

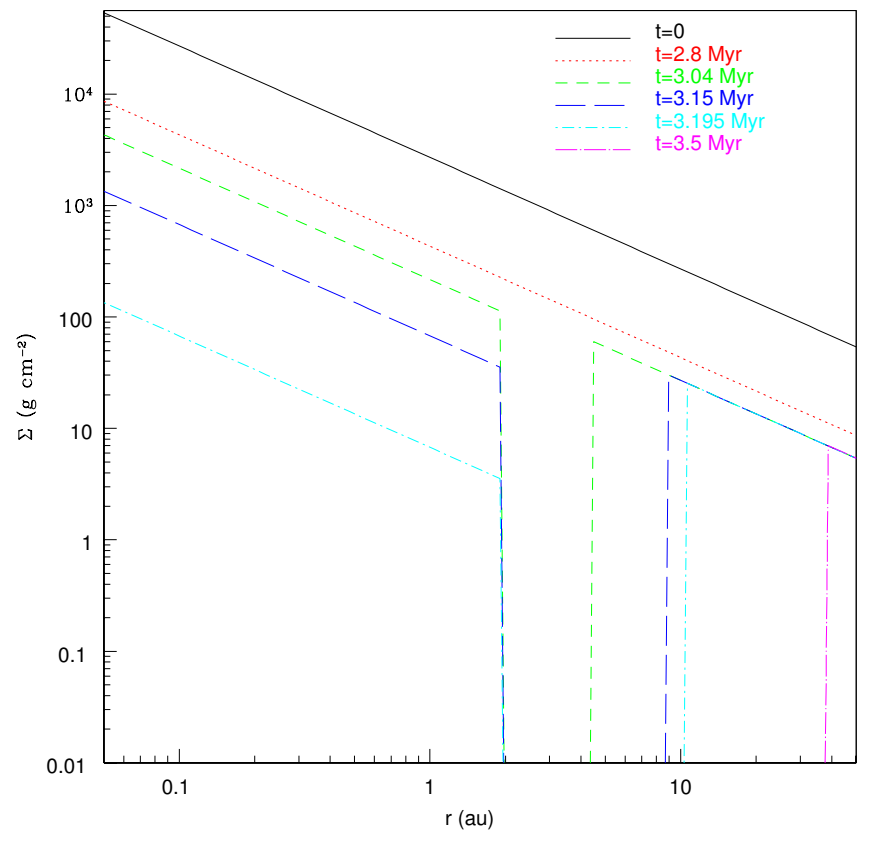

Figure 1. Surface density $\Sigma$ (in $\mathrm{g} \mathrm{cm}^{-2}$ ) versus $r$ (in au) in logarithmic scale for $t_{\text {gap }}=3 \mathrm{Myr}, t_{\nu}=0.2 \mathrm{Myr}$ and $\Sigma_{1}=2.7 \times$ $10^{3} \mathrm{~g} \mathrm{~cm}^{-2}$. The different lines correspond to the following times: $t=0$ (solid line), $t=2.8 \mathrm{Myr}$ (dotted line), $t=3.04 \mathrm{Myr}$ (shortdashed line), $t=3.15 \mathrm{Myr}$ (long-dashed line), $t=3.195 \mathrm{Myr}$ (dotted-short-dashed line) and $t=3.5 \mathrm{Myr}$ (dotted-long-dashed line). This figure is similar to fig. (9) of Owen et al. (2011).

\subsection{Migration and eccentricity damping timescales}

Tidal interaction between a planet and the disc in which it is embedded leads to a change of the planet's angular momentum (i.e. migration) and to eccentricity damping on the timescales $t_{\mathrm{mig}}$ and $t_{\mathrm{ecc}}$, respectively. The cores we consider here are small enough that they undergo inwards type I migration. In this regime, we use for $t_{\text {mig }}$ the timescale derived by Tanaka et al. (2002) and modified by Papaloizou \& Larwood (2000) to account for the effect of a finite eccentricity:

$$
\begin{gathered}
t_{\mathrm{mig}}(\mathrm{yr})=\frac{2}{2.7+1.1 n}\left(\frac{\mathrm{M}_{\odot}}{m_{p}}\right)\left(\frac{H}{r}\right)^{2} \frac{\mathrm{M}_{\odot}}{2 \pi \Sigma(1 \mathrm{au})^{2}} \\
\times\left(\frac{1 \mathrm{au}}{a}\right)^{1 / 2}\left[1+\left(\frac{e}{1.3 H / r}\right)^{5}\right]\left[1-\left(\frac{e}{1.1 H / r}\right)^{4}\right]^{-1}
\end{gathered}
$$

where $m_{p}, a$ and $e$ are the mass, semimajor axis and eccentricity of the planet, $\Sigma$ is the disc surface density of gas and $H / r$ is the disc aspect ratio at the location of the planet, and $n \equiv-d \ln \Sigma / d \ln r$. Here we have assumed that the central object is a solar mass star. Thereafter, we will take $n=1$, as in section 2.1 (see eq. 11).

For $t_{\text {ecc }}$, we adopt the timescale given by Tanaka \& Ward (2004) and again modified by Papaloizou \& Larwood (2000) to account for the effect of the eccentricity: 


$$
\begin{aligned}
t_{\mathrm{ecc}}(\mathrm{yr})=0.1\left(\frac{\mathrm{M}_{\odot}}{m_{p}}\right)\left(\frac{H}{r}\right)^{4} & \frac{\mathrm{M}_{\odot}}{2 \pi \Sigma(1 \mathrm{au})^{2}}\left(\frac{1 \mathrm{au}}{a}\right)^{1 / 2} \\
& \times\left[1+0.25\left(\frac{e}{H / r}\right)^{3}\right]
\end{aligned}
$$

where the factor of 0.1 is taken from Cresswell \& Nelson (2006) as it gives good agreement between equation (11) and the damping timescale obtained in hydrodynamical simulations.

For a planet with $e=0$ at $a=1$ au in a disc with $n=1$ and $H / r=0.05$, equations 10 and 11 can be written as:

$$
\begin{aligned}
t_{\mathrm{mig}}(\mathrm{yr}) & \simeq 6 \times 10^{5}\left[\frac{\Sigma(1 \mathrm{au})}{10^{3} \mathrm{~g} \mathrm{~cm}^{-2}}\right]^{-1}\left(\frac{m_{p}}{\mathrm{M}_{\oplus}}\right)^{-1} \\
t_{\mathrm{ecc}}(\mathrm{yr}) & \simeq 300\left[\frac{\Sigma(1 \mathrm{au})}{10^{3} \mathrm{~g} \mathrm{~cm}^{-2}}\right]^{-1}\left(\frac{m_{p}}{\mathrm{M}_{\oplus}}\right)^{-1}
\end{aligned}
$$

where $\Sigma(1 \mathrm{au})$ means that $\Sigma$ is evaluated at $1 \mathrm{au}$.

Note that radiation-hydrodynamical simulations of disc/planet interactions have shown that the corotation torque could lead to outwards migration, but that does only affect cores with masses between about 4 and $30 \mathrm{M}_{\oplus}$ (and eccentricities below $\sim 0.015$ ), larger than the cores we consider here (Paardekooper \& Mellema 2006, Kley, Bitsch \& Klahr 2009, Bitsch \& Kley 2010).

\subsection{Planet formation timescale}

Assuming that the surface density of gas at 1 au is smaller than about $10^{3} \mathrm{~g} \mathrm{~cm}^{-2}$ (see section 2.1), equation 12 indicates that migration at $\sim 1$ au only affects cores that have a mass larger than about $0.1 \mathrm{M}_{\oplus}$ (Mars's mass). Smaller mass objects would migrate on a timescale longer than the disc's lifetime, which is of a few Myr.

Cores with masses $\sim 0.1 \mathrm{M}_{\oplus}$ form through oligarchic growth on a timescale which, at $\sim 1$ au, is on the order of at least 1 Myr (see Chambers 2016 and references therein and Kobayashi \& Dauphas 2013 for the formation of Mars). Therefore, migration starts to be significant when the disc's mass is about one tenth of its initial value, i.e. $M_{50} \sim 0.01 \mathrm{M}_{\odot}$ and $\Sigma(1 \mathrm{au}) \sim 10^{2} \mathrm{~g} \mathrm{~cm}^{-2}$.

Beyond the snow line, it is expected that growth is faster, although there is still much uncertainty about building massive cores on a short timescale there (Chambers 2016). In the simulations below, we will consider cases where $1 \mathrm{M}_{\oplus}$ cores have formed on a timescale of $1 \mathrm{Myr}$ at around $5 \mathrm{au}$.

\section{NUMERICAL SIMULATIONS}

In this section, we study the evolution of a population of small cores originating from the region of the terrestrial planets, that of a population of earth mass cores originating from beyond the snow line, and finally the evolution of a mixture of both small and large cores.

\subsection{Numerical integration}

To compute the evolution of a population of cores migrating through a disc, we use the $N$-body code described in Papaloizou \& Terquem (2001) in which we have added the effect of the disc torques (see also Terquem \& Papaloizou 2007). The equations of motion are integrated using the Bulirsch-Stoer method with a timestep which is adjusted to match a prescribed accuracy (e.g., Press et al. 1992).

The equations of motion for each core are:

$\frac{\mathrm{d}^{2} \boldsymbol{r}_{i}}{\mathrm{~d} t^{2}}=-\frac{G \mathrm{M}_{\odot} \boldsymbol{r}_{i}}{\left|\boldsymbol{r}_{i}\right|^{3}}-\sum_{j=1 \neq i}^{N} \frac{G m_{j}\left(\boldsymbol{r}_{i}-\boldsymbol{r}_{j}\right)}{\left|\boldsymbol{r}_{i}-\boldsymbol{r}_{j}\right|^{3}}-\sum_{j=1}^{N} \frac{G m_{j} \boldsymbol{r}_{j}}{\left|\boldsymbol{r}_{j}\right|^{3}}+\boldsymbol{\Gamma}_{i}$,

where $G$ is the gravitational constant and $m_{i}$ and $\boldsymbol{r}_{i}$ denote the mass and position vector of core $i$, respectively. The third term on the right-hand side is the acceleration of the coordinate system based on the central star (indirect term). Acceleration due to tidal interaction with the disc is dealt with through the addition of extra forces as in Papaloizou \& Larwood (2000, see also Terquem \& Papaloizou 2007):

$$
\boldsymbol{\Gamma}_{i}=-\frac{1}{t_{\mathrm{mig}}} \frac{\mathrm{d} \boldsymbol{r}_{i}}{\mathrm{~d} t}-\frac{2}{\left|\boldsymbol{r}_{i}\right|^{2} t_{\mathrm{ecc}}}\left(\frac{\mathrm{d} \boldsymbol{r}_{i}}{\mathrm{~d} t} \cdot \boldsymbol{r}_{i}\right) \boldsymbol{r}_{i},
$$

where $t_{\mathrm{mig}}$ and $t_{\mathrm{ecc}}$ are given by equations 10 and 11 in which $m_{p}$ is replaced by $m_{i}$. Note that the timescale on which the semimajor axis decreases is $t_{\mathrm{mig}} / 2$ (e.g., Teyssandier \& Terquem 2014). As here cores never approach the star very closely, we do not include contribution from the tides raised by the star nor from relativistic effects.

Collisions between cores are dealt with in the following way: if the distance between cores $i$ and $j$ becomes less than $R_{i}+R_{j}$, where $R_{i}$ and $R_{j}$ are the radii of the cores, a collision occurs and the cores are assumed to merge. They are subsequently replaced by a single core of mass $M_{i}+M_{j}$ with the position and the velocity of the center of mass of cores $i$ and $j$.

\subsection{Initial set up}

We consider a disc which has either $\Sigma_{1}=2.7 \times 10^{3} \mathrm{~g} \mathrm{~cm}^{-2}$ or $900 \mathrm{~g} \mathrm{~cm}^{-2}$, i.e. $M_{50}=0.1$ or $0.03 \mathrm{M}_{\odot}$ initially. Note that this latter value is about twice as large as the minimum mass solar nebula (Hayashi 1981). We start with a population of $N$ cores on circular orbits in the disc midplane spread between an inner radius $r_{\text {in }}$ and an outer radius $r_{\text {out }}$. All the cores are supposed to have an identical mass density $\rho=1 \mathrm{~g} \mathrm{~cm}^{-3}$ so that $R_{i}=\left[3 m_{i} /(4 \pi \rho)\right]^{1 / 3}$. We vary $r_{\text {in }}$ and $r_{\text {out }}$ while keeping $r_{\text {out }}-r_{\text {in }}=1.5-2$ au in most of the simulations. From equation (9), the mass of dust in between those two radii is $\sim 5-10 \mathrm{M}_{\oplus}$ initially. Therefore, we will take $N \leq 100$ or $N \leq 10$ when starting with a population of cores with masses $m_{p}=0.1 \mathrm{M}_{\oplus}$ or $1 \mathrm{M}_{\oplus}$, respectively. The upper values of $N$ correspond to all the dust mass being accreted into cores, which probably leads to overestimating the number of cores or/and their masses.

As mentioned above, the disc is assumed to be truncated at an inner radius $r_{\text {cav }}=0.05$ au. At time $t=t_{\text {gap }}$, a gap opens up between $r_{10}=2$ au and $r_{20}=3 \mathrm{au}$. We take $t_{\text {gap }}=3 \mathrm{Myr}$, at which time the disc mass has de- 
creased by a factor of 10 (eq. 3] with $\eta=0.1$ ). The cores start to migrate at a time $t_{\text {start }}$ which we take to be either 1.5, 2 or 2.5 Myr. For $\Sigma_{1}=2.7 \times 10^{3} \mathrm{~g} \mathrm{~cm}^{-2}$, this corresponds to the surface density at 1 au being reduced to $1.5 \times 10^{3}, 10^{3}$ or $680 \mathrm{~g} \mathrm{~cm}^{-2}$, respectively (see eq. 3]), while for $\Sigma_{1}=900 \mathrm{~g} \mathrm{~cm}^{-2}$, it corresponds to 495,360 or $225 \mathrm{~g} \mathrm{~cm}^{-2}$, respectively. Note that, for $t$ between 0 and $t_{\text {gap }}$, the important parameters are not $t_{\text {gap }}$ and $t_{\text {start }}$ taken separately, but $t_{\text {start }} / t_{\text {gap }}$, as this is what determines the surface density in the disc when the cores start to migrate. Therefore, the same results would be obtained with $t_{\text {gap }}=1$ Myr and $t_{\text {start }}=0.5,0.7$ or $0.8 \mathrm{Myr}$.

For $t_{\nu}$, we adopt values between $2 \times 10^{4}$ and $6 \times 10^{5}$ years.

The simulations are run at least up to the time when there is no gas left in the disc in the regions were the planets are. In some cases, the simulations are run for much longer, to check the stability of the systems over Myr timescales.

\subsection{Results}

\subsubsection{Starting with small cores in the inner disc}

We start by calculating the evolution of a population of $N=50$ cores with initial masses $m_{p}=0.1 \mathrm{M}_{\oplus}$ and initially spread between 2 and 3.5 au. Figure (2) shows the evolution of the semi-major axes of these cores for $\Sigma_{1}=2.7 \times 10^{3} \mathrm{~g} \mathrm{~cm}^{-2}, t_{\mathrm{start}}=2 \mathrm{Myr}$ and $t_{\nu}=0.2 \mathrm{Myr}$. At $t=t_{\text {gap }}=3 \mathrm{Myr}$, a gap opens up between 2 and $3 \mathrm{au}$. At that point, the planet with $a>3$ au continues to migrate in. As it is in mean motion resonances (MMR) with the planets in the gap, it pushes them inwards. Migration of planets in MMR is accompanied by an increase of the eccentricities. As there is no gas left in the gap to damp the eccentricities, collisions among planets occur and resonances are destroyed. Similarly, the planets which are within 2 au continue to migrate in, and as the gas dissipates collisions occur. By the end of the simulations, only the two outermost planets are still in MMR and there is no gas left around the planets, which therefore do not migrate anymore. The three innermost planets, labelled 'A', 'B' and 'C', have $a=0.8,0.9$ and $1 \mathrm{au}$ and a mass of $0.5,0.7$ and $1.5 \mathrm{M}_{\oplus}$, respectively. The other cores left at the end of the simulation have a mass between 0.1 and $0.9 \mathrm{M}_{\oplus}$.

The eccentricities of the planets left at the end of the simulation are below 0.08 but they may increase on a longer timescale due to gravitational interactions. We note that the mutual spacing between the three innermost planets is very close to 12 times their mutual Hill radii. This has been shown by $\mathrm{Pu} \& \mathrm{Wu}(2015)$ to be the limit for stability on Gyr timescales, with smaller separations leading to instabilities. Therefore further collisions cannot be ruled out.

Figure (3) shows the difference of the longitudes of pericentre $\Delta \varpi$ for planets $\mathrm{A}$ and $\mathrm{B}$ and for planets $\mathrm{B}$ and $\mathrm{C}$ as a function of time. Before $t=3.1 \mathrm{Myr}$, these planets are in MMR. For planets $\mathrm{A}$ and $\mathrm{B}, \Delta \varpi$ librates around $180^{\circ}$, i.e. the apsidal lines are anti-aligned and conjunction occurs when one planet is near pericentre and the other near apocentre. For planets B and C, $\Delta \varpi$ librates around 0, i.e. the apsidal lines are aligned and conjunction occurs when the planets are near pericentre. At $t \simeq 3.1 \mathrm{Myr}$, a collision occurs that results in planet A merging with another core, and this destroys the MMR. At $t \simeq 3.3 \mathrm{Myr}$, planet

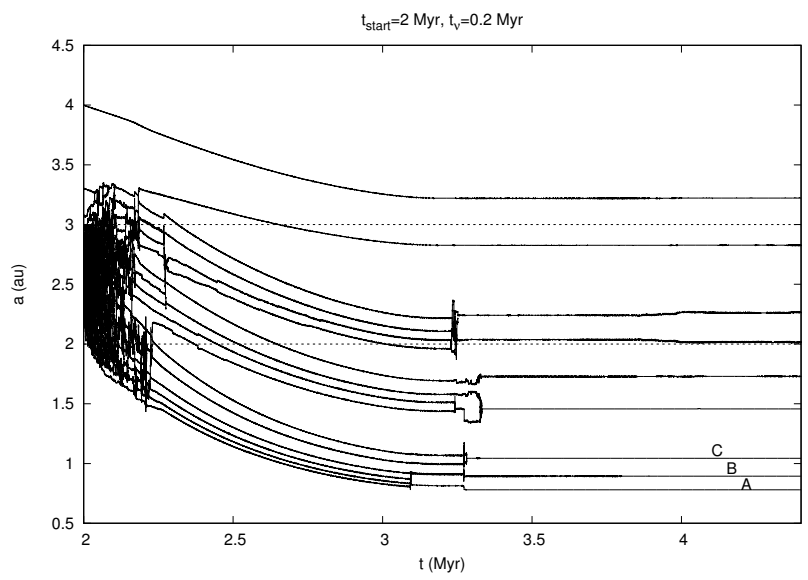

Figure 2. Evolution of the semi-major axes (in units of au) of $N=50$ cores in the system versus time (in units of Myr). Here $\Sigma_{1}=2.7 \times 10^{3} \mathrm{~g} \mathrm{~cm}^{-2}$, the cores are initially spread between $r_{\text {in }}=2$ au and $r_{\text {out }}=3.5$ au and start to migrate in at $t_{\text {start }}=2$ Myr. The gap opens up at $t_{\text {gap }}=3$ Myr between 2 and 3 au (dotted lines), and subsequently the inner disc (within $2 \mathrm{au}$ ) dissipates on the timescale $t_{\nu}=0.2 \mathrm{Myr}$. Initially, all the cores have a mass $m_{p}=0.1 \mathrm{M}_{\oplus}$. The solid lines correspond to the different cores. A line terminates just prior to a collision. The masses of the cores labelled 'A', 'B' and ' $\mathrm{C}$ ' are 0.5, 0.7 and $1.5 \mathrm{M}_{\oplus}$, respectively. The masses of the other cores left at $3.6 \mathrm{Myr}$ are between 0.1 and $0.9 \mathrm{M}_{\oplus}$.

$\mathrm{C}$ itself merges with another core. At the end of the simulation, there is no MMR among the three innermost planets (although there is some degree of dynamical coupling).

The case illustrated in figures $(2)$ and $(3)$ is very typical. All the simulations we have performed starting with $0.1 \mathrm{M}_{\oplus}$ cores and $t_{\text {start }}=2 \mathrm{Myr}$ and in which some of the cores were able to migrate below 2 au before the gap opened up ended up with a few planets between 0.5 and $\sim 1$ au. The same simulation ran with a value of $t_{\nu}$ ten times smaller, i.e. $t_{\nu}=0.02 \mathrm{Myr}$, gives qualitatively the same results. Similar results were also obtained with $t_{\text {start }}=1.5 \mathrm{Myr}$ by reducing the disc's mass (e.g., adopting $\Sigma_{1}=900$ rather than $2.7 \times 10^{3} \mathrm{~g} \mathrm{~cm}^{-2}$ ). The final masses are usually between a fraction of an Earth mass and $\sim 1 \mathrm{M}_{\oplus}$. The fact that the planets dot not migrate further down is not surprising as the migration timescale for cores with masses between 0.1 and $1 \mathrm{M}_{\oplus}$ in a disc with a surface mass density equal to a few $10^{2} \mathrm{~g} \mathrm{~cm}^{-2}$ is between 1 and $10 \mathrm{Myr}$. For $t_{\text {start }}=2 \mathrm{Myr}$, we obtained planets at the inner edge of the disc (0.05 au) only when starting with cores more massive than $0.3 \mathrm{M}_{\oplus}$. By reducing $t_{\text {start }}$ to $1.5 \mathrm{Myr}$, we could obtain planets near the edge when starting with cores more massive than $0.15 \mathrm{M}_{\oplus}$. In this case, MMR could be maintained.

In the simulation shown in figure (2), where the cores were initially spread between $r_{\text {in }}=2$ au and $r_{\text {out }}=3.5$ au, 5 cores with a total mass of $4 \mathrm{M}_{\oplus}$ reached the inner disc (within $2 \mathrm{au}$ ). The same simulation with $r_{\text {in }}=3$ au and $r_{\text {out }}=5$ au ends up with only one $1 \mathrm{M}_{\oplus}$ core slightly below $2 \mathrm{au}$, all the others being left at larger distances from the star. On the other hand, by reducing $r_{\text {in }}$ and $r_{\text {out }}$ to 1.5 and $3 \mathrm{au}$, respectively, we obtain 8 cores with a total mass of $4.6 \mathrm{M}_{\oplus}$ between 0.5 and $2 \mathrm{au}$, the most massive core having a mass of $1 \mathrm{M}_{\oplus}$. Again, the orbits of these cores are expected 

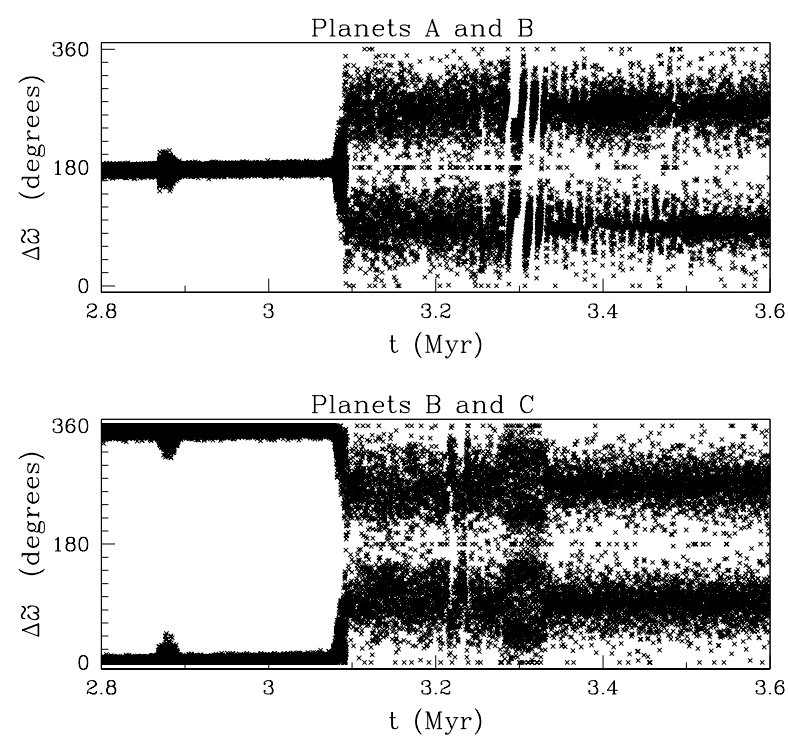

Figure 3. Difference of the longitudes of pericentre $\Delta \varpi$ (in degrees) for planets $\mathrm{A}$ and $\mathrm{B}$ (upper plot) and for planets B and $\mathrm{C}$ (lower plot) shown in figure 22 versus time (in units of Myr). Before $t=3.1 \mathrm{Myr}$, these planets are in MMR with $\Delta \varpi$ librating around either $180^{\circ}$ or 0 . A collision during which planet A merges with another core occurs at $t \simeq 3.1 \mathrm{Myr}$ and destroys the resonances. At the end of the simulation, there is no MMR among the three innermost planets.

to evolve and further collisions to occur on a timescale longer than that of the simulation.

When the cores do not migrate significantly, either because the initial disc is not massive enough (low $\Sigma_{1}$ ) or migration starts late, after the disc's mass has decreased down to low values (large $t_{\text {start }}$ ), evolution is very much like what is obtained in in situ formation models. There is not enough gas to damp the eccentricities, so collisions occur and a few planets with masses at most between 1 and $2 \mathrm{M}_{\oplus}$ form without significant inward migration.

\subsubsection{Starting with heavier cores beyond the snow line}

So far, we have ignored in the simulations heavier cores that may be delivered to the inner parts from further away in the disc. Whether these cores will be present or not depends on wether they can form on a timescale of 1 Myr. Let us assume this the case, and there is a population of $1 \mathrm{M}_{\oplus}$ cores that form within $\sim 1$ Myr beyond the snow line at around $5 \mathrm{au}$ (see Chambers 2016 for models). According to equation 10 , the migration timescale of these cores at 5 au would be the same as that of $0.1 \mathrm{M}_{\oplus}$ cores at $1 \mathrm{au}$, and therefore we choose the same value of $t_{\text {start }}=2 \mathrm{Myr}$ as in the simulations with smaller cores presented above (we also keep $t_{\nu}=0.2 \mathrm{Myr}$ ). In figure (4), we show the evolution of 10 cores with initial mass of $m_{p}=1 \mathrm{M}_{\oplus}$ and initially spread between 5 and $6.5 \mathrm{au}$, for both $\Sigma_{1}=2.7 \times 10^{3}$ (upper plot) and $900 \mathrm{~g} \mathrm{~cm}^{-2}$ (lower plot). As pointed out above, if these cores had formed in situ they would have used all the dust initially present in the disc at these locations. Alternatively, they could have formed further away and migrated in, although it takes more time to form cores at larger distances from the star. As seen on the figure, these cores grow through collisions and migrate in while maintaining MMR. When the gap opens up, in the case where $\Sigma_{1}=2.7 \times 10^{3} \mathrm{~g} \mathrm{~cm}^{-2}$, some of the cores have reached the inner parts of the disc below 2 au while the other cores are in the gap. The depletion of gas leads to eccentricity growth and further collisions occur, leaving at the end of the simulation two cores with masses of 3 and $6 \mathrm{M}_{\oplus}$ between 1 and 2 au and one core with a mass of $1 \mathrm{M}_{\oplus}$ near $3 \mathrm{au}$. In the case of a less massive disc, i.e. $\Sigma_{1}=900 \mathrm{~g} \mathrm{~cm}^{-2}$, none of the cores reach the gap before it opens up. Here, by the end of the simulation, a $1 \mathrm{M}_{\oplus}$ core has been scattered to large distance $(\sim 11 \mathrm{au})$ while two cores with masses of 7 and $2 \mathrm{M}_{\oplus}$ are left at 4 and $\sim 3$ au. In that case, evolution is very much like what is obtained with in situ models.

As can be seen from the figure, the systems are stable after the last collisions occur and until 6 Myr. The eccentricities, although large, vary smoothly and in a periodic way. However, further collisions over much longer timescales cannot be ruled out.

Cores with a mass of a few $\mathrm{M}_{\oplus}$ could be obtained at smaller distances from the star by starting the migration at earlier times.

\subsubsection{Starting with a mixture of small and heavier cores}

If we now start with both small cores $\left(m_{p}=0.1 \mathrm{M}_{\oplus}\right)$ at around $2 \mathrm{au}$ and heavier cores $\left(m_{p}=1 \mathrm{M}_{\oplus}\right)$ at around $5 \mathrm{au}$, the evolution can be predicted on the basis of the results presented above. The small cores evolve as described above, and always end up forming planets with masses at most between 1 and $2 \mathrm{M}_{\oplus}$ at locations that depend on how fast they have migrated, but usually between 0.5 and $2 \mathrm{au}$. If the heavier cores are able to catch up, they may sweep some of the smallest ones on their way in and the final system has a few cores of several earth masses below $2 \mathrm{au}$, mixed with less massive planets. On the other hand, the heavier cores may not be able to migrate below the inner edge of the gap (e.g., for low values of $\Sigma_{1}$ or if the heavy cores have large $\left.t_{\text {start }}\right)$, in which case they will also evolve mostly in situ to form a few massive cores beyond 4 au or so.

This is exactly what we have found in simulations starting with 50 small cores $\left(m_{p}=0.1 \mathrm{M}_{\oplus}\right)$ between 2 and $5 \mathrm{au}$ together with 5 or 10 heavier cores $\left(m_{p}=1 \mathrm{M}_{\oplus}\right)$ between 5 and $6.5 \mathrm{au}$. We considered $\Sigma_{1}=2.7 \times 10^{3}$ or $900 \mathrm{~g} \mathrm{~cm}^{-2}$ and, as above, $t_{\text {start }}=2 \mathrm{Myr}$ and $t_{\nu}=0.2 \mathrm{Myr}$. The case $\Sigma_{1}=2.7 \times 10^{3} \mathrm{~g} \mathrm{~cm}^{-2}$ is illustrated in figure $(5)$. The simulation has been stopped after $8 \mathrm{Myr}$ but here of course more collisions are going to occur on longer timescales. The same simulation with $\Sigma_{1}=900 \mathrm{~g} \mathrm{~cm}^{-2}$ does not show any significant migration. In that case, the cores evolve mostly in situ, and after the disc dissipates there is no core below $1.8 \mathrm{au}$.

\section{SUMMARY AND DISCUSSION}

In this paper, we have studied the outcome of core migration in transition discs produced by photoevaporation. The initial surface mass density in discs at around 1 au is at 

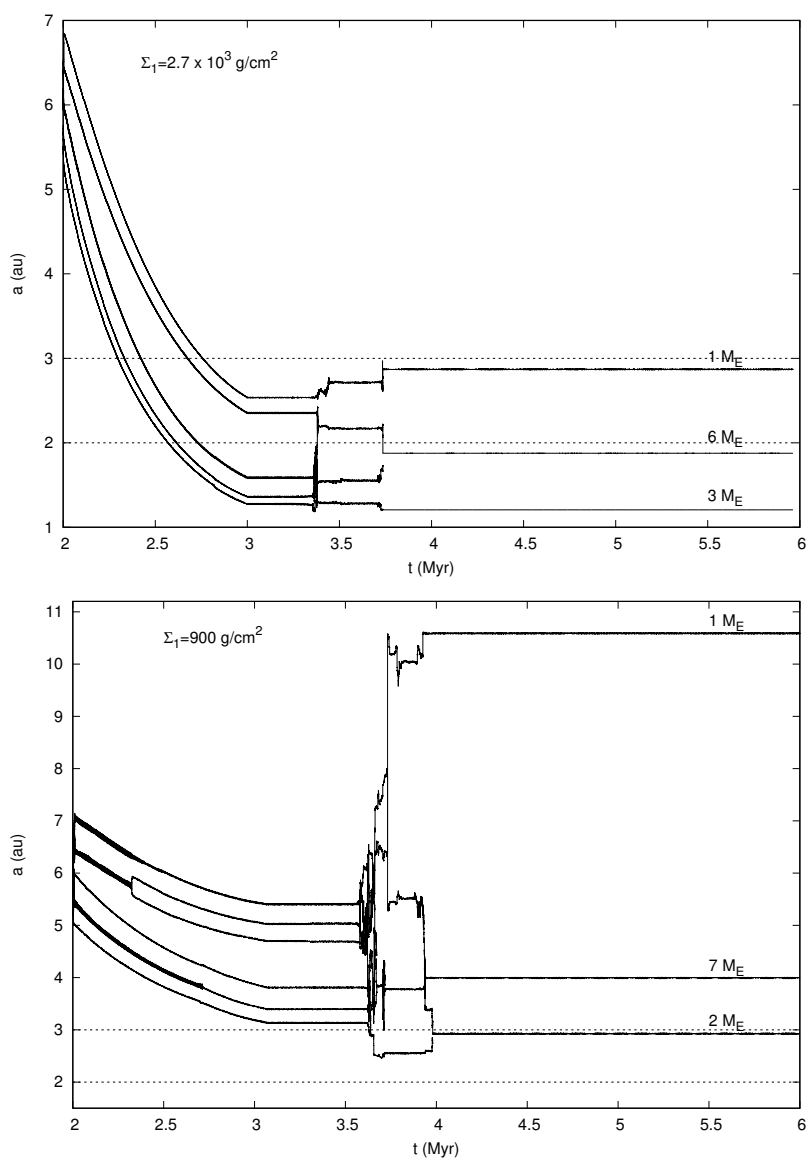

Figure 4. Same as figure 2 but for $N=10, m_{p}=1 \mathrm{M}_{\oplus}$, $r_{\text {in }}=5 \mathrm{au}, r_{\text {out }}=6.5 \mathrm{au}$ and $\Sigma_{1}=2.7 \times 10^{3} \mathrm{~g} \mathrm{~cm}^{-2}$ (upper plot) or $900 \mathrm{~g} \mathrm{~cm}^{-2}$ (lower plot). Collisions between initial cores occur very early in the simulations so the 10 cores are not visible on the plots. Also heavy lines indicate several cores which oscillate around the same semi-major axis. The numbers above the lines at the end of the simulations indicate the mass of the cores left in $\mathrm{M}_{\oplus}$.

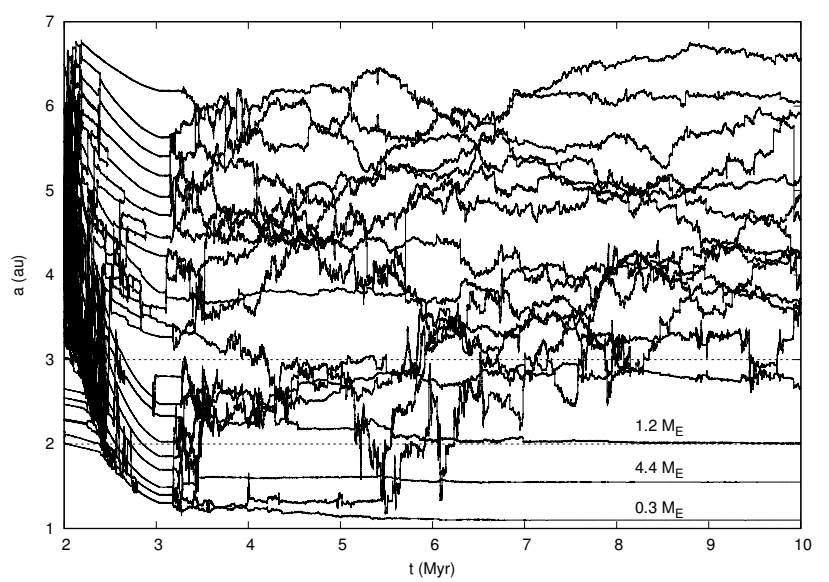

Figure 5. Same as figure 2 but here there are initially 50 cores with $m_{p}=0.1 \mathrm{M}_{\oplus}$ between 2 and 5 au and 5 cores with $m_{p}=$ $1 \mathrm{M}_{\oplus}$ between 5 and 6.5 au. The numbers above the lines at the end of the simulation indicate the mass of the cores left in $\mathrm{M}_{\oplus}$. most on the order $10^{3} \mathrm{~g} \mathrm{~cm}^{-2}$, and it decreases with time. Therefore, planetary cores that form at this distance from the star migrate on a timescale smaller than the disc lifetime only if their mass is larger than about $0.1 \mathrm{M}_{\oplus}$. As it takes at least about 1 Myr to form such cores at around $1 \mathrm{au}$, migration of cores forming below the snow line starts to be relevant only when the disc is at least 1 Myr old. Similarly, if the surface density varies as $r^{-1}$, cores at $\sim 5$ au have a migration timescale shorter than the disc lifetime only when their mass reaches about $1 \mathrm{M}_{\oplus}$. Assuming these cores form on a timescale of about $1 \mathrm{Myr}$, they will also start to migrate significantly when the disc is about 1 Myr old.

In low mm flux transition discs, a gap is believed to open up at around 2-3 au due to $\mathrm{X}$-ray photoevaporation when the disc is a few Myr. The disc's inner parts are subsequently accreted onto the central star as a result of viscous evolution, while the outer edge of the gap recedes under the effect of photoevaporation. In this context, we find that $\sim 0.1 \mathrm{M}_{\oplus}$ cores that form within $\sim 1$ Myr between 1-4 au end up forming a system of a few planets with masses between a fraction of an Earth mass and $1.5 \mathrm{M}_{\oplus}$ at most. In general, these cores do not migrate down further than $\sim 0.5 \mathrm{au}$, and may even evolve in situ in low mass discs. They are not in mean motion resonances. Such resonances, which appear during the migration phase, are usually destroyed when the gas dissipates (as previously found by Cossou et al. 2014 and Coleman \& Nelson 2016). It is difficult to form planets at smaller radii, unless we start with heavier cores or start migrating the cores earlier (as, e.g., in Terquem \& Papaloizou 2007). In both cases, it would require a more efficient planet formation process than envisioned here. If $\sim 1 \mathrm{M}_{\oplus}$ cores can form beyond the snow line also within $\sim 1 \mathrm{Myr}$, they migrate on the same timescale as the smaller cores and, depending on the surface density in the disc, they may or may not reach the inner parts of the disc. After the gap has opened up, the inner parts of the disc become isolated from the outer parts, and no more material is delivered there.

We have found that, in a disc with an initial surface mass density of about $10^{3} \mathrm{~g} \mathrm{~cm}^{-2}$ at $1 \mathrm{au}$, it was possible to obtain a system of cores similar to that of the Solar system, with a few planets with masses of a few tenths of an earth mass to an earth mass in the terrestrial zone and a more massive core a little bit further away (fig. 22 and lower panel of fig. 4]). However, as the photoevaporation model predicts that the outer edge of the gap is receding rather fast, it is difficult to envision within this model how the massive core could accrete a gaseous envelope to become a giant planet. Such a system of low mass planets is similar to those obtained by Cossou et al. (2014) and Coleman \& Nelson (2016) in their models with inefficient migration.

The exact parameters that should be used in such simulations are of course not known, and different outcomes could be produced by changing them. However, the point of this paper is to show that, by adopting reasonable parameters that fit the observations and the current understanding of planet formation and migration, systems of cores/planets can be obtained where all the planets do not end up on resonant chains near the disc's inner edge and delivery of material to the inner parts from the outer parts of the disc can be avoided. To that extent, the type of systems that we obtain resembles more our Solar system than the systems observed by Kepler, which is biased towards detecting plan- 
ets on very tight orbits. Our results predict that low $\mathrm{mm}$ flux transition disc may harbour terrestrial planets in the habitable zone.

Note that, as collisions between cores occur until rather late in the disc's evolution, dust may be produced at distances below 1 or 2 au from the central star even after the disc's inners parts have been accreted. In that case, the disc would not appear as transition disc.

This study suggests that low mm flux transition discs may not be able to form giant planets before $\mathrm{X}$-ray photoevaporation opens up a gap. These discs would then form planetary systems of the type obtained in this study: predominantly low mass planets at around 1 au with possibly more massive cores further away. High mm flux transition discs, by contrast, are associated with more massive stars and may form giant planets of a few Jupiter masses, massive enough to open up a gap, before photoevaporation could proceed.

\section{REFERENCES}

Andrews S. M., Wilner D. J., Hughes A. M., Qi C., Dullemond C. P., 2010, ApJ, 723, 1241

Bitsch B., Kley W., 2010, A\&A, 523, 30

Chambers J. E., 2016, ApJ, 825, 63

Coleman G. A. L., Nelson R. P., 2014, MNRAS, 445, 479

Coleman G. A. L., Nelson R. P., 2016, MNRAS, 457, 2480

Cossou C., Raymond S. N., Hersant F., Pierens A., 2014, A\&A, 569,56

Cresswell P., Nelson R. P., 2006, A\&A, 450, 833

Haghighipour N., 2013, AREPS, 41, 469

Hansen B. M. S., Murray N., 2013, ApJ, 775, 53

Hartmann L., Calvet N., Gullbring E., DAlessio P., 1998, ApJ, 495,385

Hayashi C., 1981, Prog. Theor. Phys. Suppl., 70, 35

Kley W., Bitsch B., Klahr H., 2009, A\&A, 506, 971

Kobayashi H., Dauphas N., 2013, Icarus, 225, 122

Lissauer J. J., 1993, ARA\&A, 31, 129

Owen J. E., 2016, PASA, 33, 5

Owen J. E., Clarke C. J., 2012, MNRAS, 426, 96

Owen J. E., Ercolano B., Clarke C. J., 2011, MNRAS, 412, 13

Paardekooper S.-J., Mellema G., 2006, A\&A, 459, L17

Papaloizou J. C. B., Larwood J. D., 2000, MNRAS, 315, 823

Papaloizou J. C. B., Terquem C., 2001, MNRAS, 325, 221

Pu B., Wu Y., 2015, ApJ, 807, 44

Raymond S. N., Cossou C., 2014, MNRAS, 440, L11

Schlichting H. E., 2014, ApJ, 795, 15

Shakura N. I., Sunyaev R. A., 1973, A\&A, 24, 337

Tanaka H., Takeuchi T., Ward W. R., 2002, ApJ, 565, 1257

Tanaka H., Ward W. R. 2004, ApJ, 602, 388

Terquem C., Papaloizou J. C. B., 2007, ApJ, 654, 1110

Teyssandier J., Terquem C., 2014, MNRAS, 443, 568

Thommes E. W., Duncan M. J., Levison H. F. 2003, Icarus, 161, 431

Wetherill G. W., 1988, in Mercury, ed. F. Vilas, C. R. Chapman, M. S. Matthews, p. 670 (Tucson: Univ. Ariz. Press) 